Chapman University

Chapman University Digital Commons

$7-2020$

\title{
Mini-Logger- A Wearable Inertial Measurement Unit (IMU) for Postural Sway Analysis
}

Michael Pollind

Rahul Soangra

Follow this and additional works at: https://digitalcommons.chapman.edu/pt_articles

Part of the Other Rehabilitation and Therapy Commons, and the Physical Therapy Commons 


\section{Mini-Logger- A Wearable Inertial Measurement Unit (IMU) for Postural Sway Analysis}

\section{Comments}

This is a pre-copy-editing, author-produced PDF of an article presented at the 2020 42nd Annual International Conference of the IEEE Engineering in Medicine \& Biology Society (EMBC). The definitive publisher-authenticated version is available online at https://doi.org/10.1109/EMBC44109.2020.9175167.

\section{Copyright}

(c) 2020 IEEE. Personal use of this material is permitted. Permission from IEEE must be obtained for all other uses, in any current or future media, including reprinting/republishing this material for advertising or promotional purposes, creating new collective works, for resale or redistribution to servers or lists, or reuse of any copyrighted component of this work in other works. 


\title{
Mini-Logger- A Wearable Inertial Measurement Unit (IMU) for Postural Sway Analysis
}

\author{
Michael L. Pollind, and Rahul Soangra*- IEEE Member
}

\begin{abstract}
Postural instability assessment is an important tool in fall risk analysis and for the timely intervention of falls to reduce or prevent fall injuries. Traditionally fall risk is measured through postural sway assessment and is collected through forceplates by mapping Center of Pressure (COP) excursions or using motion analysis camera system for marker sway trajectories. However, both of these systems are expensive and lack portability to their usage in clinical environments. In this study, we developed a novel wearable low-cost MEMS inertial sensor and validated its usage for human postural sway assessment in standing posture with eyes open/closed, vibration/no vibration, and proprioception /low proprioception conditions. The two objectives of this study were: 1) To develop and validate an Inertial Measurement Unit (IMU) for sway analysis 2) To determine the feasibility of the system in detecting human postural imbalances such as reduced proprioception or presence of stochastic resonance induced through subthreshold vibrations on the feet. The novel IMU was tested for sway against infra-red marker on a specialized platform with 4-degrees of freedom. Many parameters of postural sway such as sway velocity, Root Mean Square (RMS), and sway path length could successfully detect subtle postural changes due to varying proprioceptive and sub-threshold vibration conditions. We found agreement in sway signal determinism from the two methods.
\end{abstract}

Clinical Relevance - This wearable sensor technology has potential to determine balance in reliable, easy and accurate way in clinical environments.

\section{INTRODUCTION}

Maintenance of postural stability requires integration of three sensory inputs from visual, vestibular, and somatosensory systems to maintain the center of gravity (COG) with in the base of support. Postural balance is dependent on the dynamic interaction between sensory inputs and motor processes. The maintenance of static and dynamic postural balance involves the integration of sensory inputs through a dynamic process called sensory reweighting [1]. Therefore, the normal functioning of sensory and proprioceptive system is essential for successful postural control in any static and dynamic conditions. Any slight impairments of sensory system could lead to postural control deficits, thereby increase fall risk. Older people have diminished tactile sensation of the sole and ankle proprioception specifically with diabetic neuropathy. This loss of sensory inputs influences the feedback received by the central nervous system to correct postural responses, leading to dependence on visual and vestibular information [2]. Thus, somatosensory inputs impair balance. A mechanism to

\footnotetext{
*Research supported by Crean College of Health and behavioral Sciences, Chapman University.

Michael Pollind works with Prospect Medical, Orange, CA 92618 USA

Rahul Soangra, Assistant Professor, is with Chapman University, Orange, CA 92618 USA. He is an Assistant Professor in Department of Physical
}

amplify the sensory inputs to an individual with diminished somatosensory inputs could potentially restore balance and decrease fall risk. Stochastic resonance is a phenomenon in which white noise is introduced to boost a underlying subthreshold signal to cross a receptor's sensory threshold; therefore, improving the system's sensitivity to detect the proprioceptive information [3]. Stochastic resonance is addition of a weak input signal of noise to an existing weak signal resulting in to a stronger resultant signal that can surpass the sensory threshold of a sensory neuron's receptor [4]. Appropriate signal reception allows useful sensory information to pass to the central nervous system and allowing for remediating responses and balance restoration.

A few research studies have already supported the use of stochastic resonance to improve postural control in the elderly[5]. Other studies have highlighted clinical and biomechanical effects of stochastic resonance on the plantar surface of the foot in diabetic patients and older people[2, 6]. Application of noise through vibrating actuators or motors embedded in shoe insoles to improve balance [7] have been demonstrated in few studies. Stochastic resonance has been studied with outcome measures such as center of pressure via forceplates (postural sway), postural sway via motion analysis, and other temporal and spatial gait parameters via motion analysis $[8,9]$. These studies have suggested that subthreshold noise improved balance control, tactile sensation[10,11], and vibration sensation [2]. In this study we aim to develop and investigate the determinism of sway signals of a new wearable sensor device developed in our laboratory (Mini-Logger) to available gold standard camera-based motion capture system with specialized sway platform with 4 Degrees of Freedom (DOF). We also investigated indiathe sensitivity of the novel wearable sensor on human postural sway at different visual, proprioceptive and vibratory conditions.

\section{MATERIALS AND METHODS}

\section{A. Development of IMU}

Currently, many of the sensors available in the market for acceleration and gyroscopic data are expensive and cost upwards of 1000 USD. We designed a new inertial measurement unit (IMU) named Mini-Logger to be a more affordable IMU for human postural sway analysis. It is a small wearable sensor that was designed to collect linear acceleration and gyroscopic moments at a variable frequency up to $100 \mathrm{~Hz}$; it has capability to store data onto a removable micro SD card. The novel Mini-Logger follows a similar

Therapy and also an adjunct in Department of Electrical Engineering and Computer Sciences, Chapman University, Orange, CA 92618 USA (e-mail: soangra@chapman.edu; Phone: +1-714-516-6160; Fax:+1-949-206-0012). 
construction to an Arduino Pro Mini - containing a microprocessor (ATmega-328-AU), power supply (3.3V), and 6 programmable pin headers. The data acquisition component is an ICM-20498, a 9 axis (3-axis gyroscope, 3-axis accelerometer, and 3-axis magnetometer) motion tracking device. For the purposes of this sensor, only 6 axes are used. The ICM-20498 was chosen because of the compact size of the chip and a very low power requirement in comparison to other motion tracking devices.

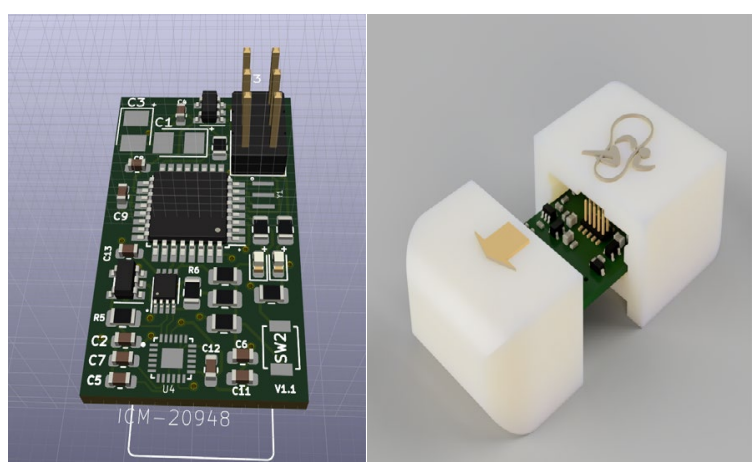

Figure 1. Design and fabrication of IMU, named Mini-Logger

While the lower power consumption is an advantage for extending the battery life, it does create a challenge of connecting the chip to the rest of the board. The ICM-20498 has an operating voltage of 1.81 volts whereas the other components use 3.3 volts. In order to combat this problem, an I2C bridge (2004PCA9306DCUR) was added to convert the higher voltage of $3.3 \mathrm{~V}$ to $1.8 \mathrm{~V}$, allowing the ICM-20498 to draw power directly from the board instead of an additional external power source. The outer casing for the Mini-Logger was designed using Autodesk Fusion 360. A simple snap-fit design was used to secure the sensor inside the enclosure. Complete details about the fabrication are available at https://github.com/GaitRehabilitation/mini_data_capture.

\section{B. Validation of IMU}

The performance of Mini-Logger was tested against a laboratory-based gold-standard infra-red motion capture camera system for assessing sway. The sensor was mounted appropriately on a treadmill platform with 4 Degrees of Freedom (DOF) Fig. 2a. The repetitive systematic sway produced by the platform was an ideal setup for validation of sway measured by the two systems i) Mini-Logger and ii) Infra-red Marker. The platform (GRAIL, Motek medical, netherlands) could generate 10 degrees of pitch and $5 \mathrm{~cm}$ of translation. Two sinusoidal equations were generated for pitch and translation excursions as shown in equations 1 and 2 below.

$$
\begin{aligned}
& \text { Pitch } \\
& =\text { percent }_{\text {magnitude }} * 10 \\
& * \sin \left(\text { percent }_{\text {frequency }} * \text { TIME }\right) \\
& \text { Translation } \text { percent }_{\text {magnitude }} * 0.05 \\
& * \sin \left(\text { percent }_{\text {frequency }} * \text { TIME }\right)
\end{aligned}
$$

The Percent magnitude and Percent $_{\text {frequency }}$ ranged from $100 \%$, $80 \%, 40 \%$ and $20 \%$ of the total range of motion. For instance, pitch (maximum amplitude 10 degrees) had an amplitude of $\pm 10, \pm 8, \pm 4, \pm 2$ degrees and translation of $\pm 5, \pm 4, \pm 2, \pm 1 \mathrm{~cm}$ of horizontal motion. The movement data was filtered using zero-lag dual low-pass Butterworth filter of $4^{\text {th }}$ order and cutoff frequency as $8 \mathrm{~Hz}$. Nonlinear variability analysis techniques such as Recurrent Quantification Analysis (RQA) was used to validate recurrence and determinism between signals from the infra-red marker and Mini-Logger, which were generated from a single movement source (platform here Fig. 2a). The platform is a part of the Gait Research Analysis and Interactive Laboratory (GRAIL, Motek Medical, Netherland).

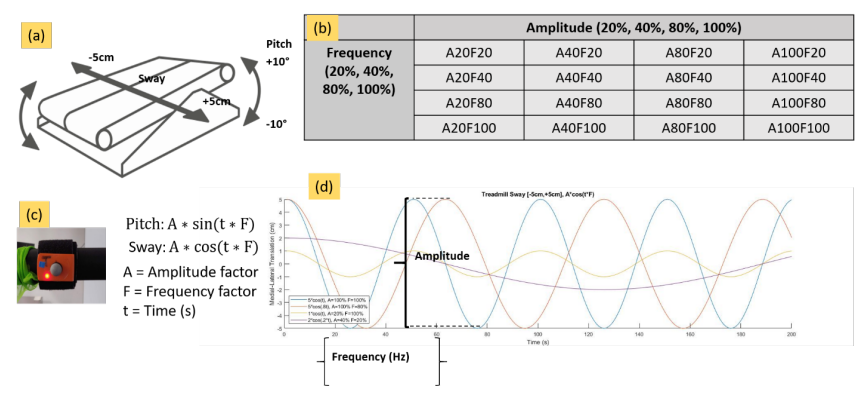

Figure 2. (a) Platform with various degrees of freedom to generate sway (b) Various amplitude and frequency of sway generated (c) Sensor and Infrared Marker (d) Sinusoidal waves showing amplitude and frequency generated

RQA is used to quantify the duration and number of recurrences in the time series. The time delay and the number of dimensions to embed the signal were computed [12]. Average Mutual Information (AMI) was used to find optimal time delay for embedding. Phase space plots were constructed utilizing axeses quantified by $((\mathrm{x}(\mathrm{t}), \mathrm{x}(\mathrm{t}+\tau))$ where $\tau$ is a constant that shifts the signal by some factor from original $\mathrm{x}(\mathrm{t})$ and $\mathrm{x}(\mathrm{t}+\tau)[12]$. This can be resolved by a nonlinear generalization of the auto-correlation function and is given by equation below[12]

$$
I(x(t), x(t, \tau))=\sum_{i, j}\left(p_{i, j}(\tau) \log \left(\frac{p_{i, j}(\tau)}{p_{i} p_{j}}\right)\right.
$$

False Nearest Neighbors (FNN) is used to find the optimal lag of the signal[12]. A state-space is reconstructed by embedding the original one-dimensional time series utilizing time delayed signals. This can be described by the original time series $\mathrm{X}(\mathrm{t})$ and follows:

$$
y(t)=(x(t), x(t+\tau), \ldots ., x(t+(D-1) \tau))
$$

Where $t$ and $\tau$ are integers that are indexed on the sample data [12]. The embedding dimension ( $\mathrm{m}$ ) can be estimated by examining the distance between points in phase space. If the embedding distance between the two neighbors changes appreciably then the neighbors are considered false neighbors and are true neighbors otherwise[12]. This is continued until the number of FNN drops to 0 or minimum[12]. Recurrent Quantitative Analysis (RQA) plots: The diagonal structure in the recurrence plots (RP) tends to suggest that the time series function has period to quasi-periodic recurrent structures[13]. This regularity in the structure suggests that the movement signal source repeated at a fixed interval[13]. However more quasi-period systems will have variations in the distance between diagonal segments[13]. The source for the signal was a fixed sinusoidal wave so this is expected when reviewing the recurrence plot data. Fig. 3 represents how the recurrence plot can be constructed from time series signals with known delay and dimensions. Recurrence Rate (RR): The distance between diagonal lines in RP space is referred to as the recurrence rate $(\mathrm{RR}) . \mathrm{P}(\mathrm{l})$ is defined as the number of points 
sampled on the diagonal line segment[13], or the histogram of the lengths 1 of the diagonal lines. The main diagonal for the $\mathrm{RP}$ plot is fixed across all the trials and is excluded when examining the $R R$. $R R_{\tau}$ is described by the equation above where $\tau=0$ is the main diagonal and $\tau>0$ is above the main diagonal and $\tau<0$ is the below the diagonal[13].

$$
R R_{\tau}=\sum_{i=1}^{N-\tau}\left(R_{i, i+1}\right)
$$

This measure directly relates to the likely hood that an event will occur again given some delay $\tau$. This can be generalized as a higher-order autocorrelation function as it is dependent on $\tau$ [13]. Determinism (DET) is defined as the percentage of recurrence points which form the diagonal lines

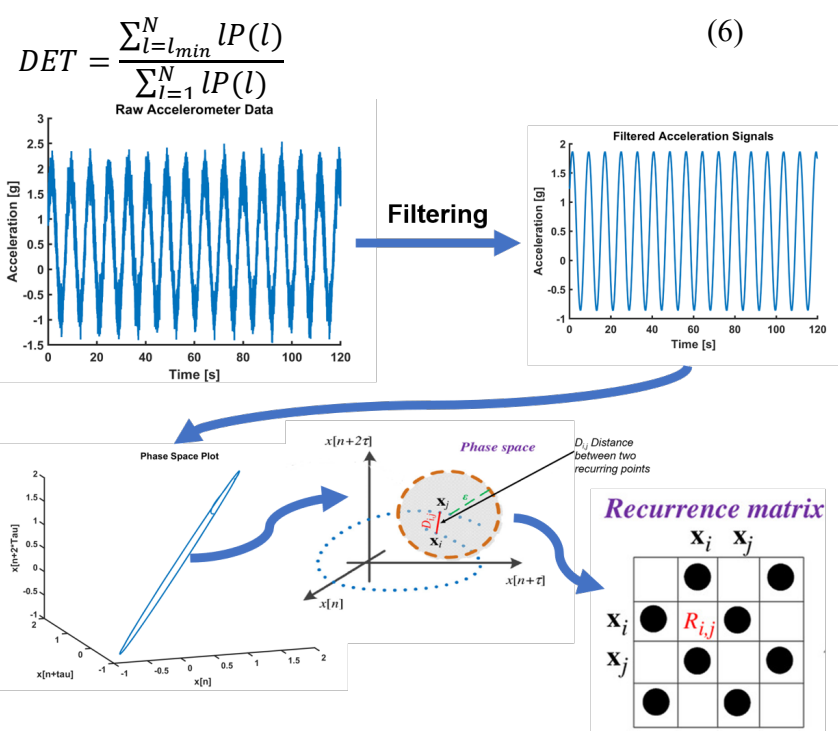

Figure 3. Accelerometer signals from the platform sway were filtered for highfrequency noise and phase space reconstruction was performed for recurrence quantification

Experimental Protocol: Ten healthy subjects (5 males and 5 females, age: $27.3 \pm 2.7$ years, $68 \pm 5.6 \mathrm{Kg}, 183 \pm 4 \mathrm{~cm}$ ) participated in this study approved by Chapman University IRB. Postural balance for each subject was assessed under a variety of conditions in randomized order, adjusting variables of visual input, proprioceptive input, and frequency, for a total of 12 conditions. For vision, participants were instructed to keep eyes open (EO) and focus on a target five meters in front of them or to keep eyes closed (EC). Proprioceptive input was modified by adjusting surface conditions, having the participant stand directly on the solid vinyl surface or on STEPRIGHTR stability trainers (Fig. 4a, 4b, 4c). Varying frequency input was provided with tactors placed on the dorsal surface of $1 \mathrm{st}$ metatarsal on bilateral feet $(0 \mathrm{~Hz}, 220 \mathrm{~Hz}$, $260 \mathrm{~Hz}$ ). The amplitude of frequency was adjusted to subthreshold levels so that subjects were unable to perceive vibration. Under each condition, subjects were instructed to stand on a force plate with feet shoulder width apart for a total of 60 seconds. Both motion capture systems infra-red marker and Mini-Logger were placed on subject during trials. During the data collection, the students were asked to stand in place for one minute and had to undergo one of 12 conditions, shown in Table I below. For the vibration tactor trials, a tactor was attached to the first metatarsal (Fig. 4c and 4d) and the frequency was changed in order to see if the balance of the student would change. For the trials without and with StepRight system were used to lower the points of contact of the foot (Fig. 4b).

TABLE I. DIFFERENT CONDITIONS OF POSTURAL STANDING

\begin{tabular}{|l|c|c|l|}
\hline Condition \# & $\begin{array}{c}\text { Tactor } \\
\text { Frequency }\end{array}$ & $\begin{array}{c}\text { Eyes } \\
\text { Open/Clo } \\
\text { sed }\end{array}$ & Surface Condition \\
\hline 1 & $0 \mathrm{~Hz}$ & $\mathrm{EO}$ & No StepRight \\
\hline 2 & $0 \mathrm{~Hz}$ & $\mathrm{EO}$ & StepRight \\
\hline 3 & $0 \mathrm{~Hz}$ & $\mathrm{EC}$ & No StepRight \\
\hline 4 & $0 \mathrm{~Hz}$ & $\mathrm{EC}$ & StepRight \\
\hline 5 & $220 \mathrm{~Hz}$ & $\mathrm{EO}$ & No StepRight \\
\hline 6 & $220 \mathrm{~Hz}$ & $\mathrm{EO}$ & StepRight \\
\hline 7 & $220 \mathrm{~Hz}$ & $\mathrm{EC}$ & No StepRight \\
\hline 8 & $220 \mathrm{~Hz}$ & $\mathrm{EC}$ & StepRight \\
\hline 9 & $260 \mathrm{~Hz}$ & $\mathrm{EO}$ & No StepRight \\
\hline 10 & $260 \mathrm{~Hz}$ & $\mathrm{EO}$ & StepRight \\
\hline 11 & $260 \mathrm{~Hz}$ & $\mathrm{EC}$ & No StepRight \\
\hline 12 & $260 \mathrm{~Hz}$ & $\mathrm{EC}$ & StepRight \\
\hline (a) & & (c) & \\
\hline
\end{tabular}

Figure 4. (a) StepRight for reduced proprioceptive inputs (b) inflated StepRight (c) Tactors attached at first metatarsal when using StepRight and standing on forceplate (d) standing without StepRight on forceplate with tactors

\section{RESULTS}

Data Analysis: Data was analyzed using multivariable analysis of variance (MANOVA) and JMP 14 Pro software. The significance level was set at 0.05 for all analyses. We found RMS of signals when using StepRight (reduced proprioceptive condition) at $0 \mathrm{~Hz}(\overline{\mathrm{x}}=0.035)$ was significantly higher ( $\mathrm{p}=0.0155)$ when compared to the condition when using StepRight at $220 \mathrm{~Hz}(\overline{\mathrm{x}}=0.026)$. However, no differences were observed at $260 \mathrm{~Hz}$. The sway velocity increased significantly $(p<0.01)$ when using StepRight in the eyes-closed condition. Similarly, the sway path increased significantly $(\mathrm{p}<0.01)$ in the eyes-closed condition using StepRight. We found determinism recurrence rate were comparable in both of the systems.

\section{DISCUSSION}

The primary objective of this study was to build a wearable system (Mini-Logger), validate it with laboratory-based goldstandard laboratory equipment and evaluate its relative sensitivity for sway measures to different proprioceptive and visual conditions in human subjects. Mini-Logger was designed and fabricated (Fig. 1) and was validated for sway analysis on a 4-DOF platform (Fig. 2) available commercially for virtual reality-based gait analysis. The movement was generated using two sinusoidal patterns generated in pitch and translation. The Mini-Logger and Vicon camera system both sampled at the sampling frequency of $100 \mathrm{~Hz}$. It is practically difficult to align the directions (axes) of global origin (for camera system) and sensitive directions of Mini-Logger, we based our analysis on resultant 3-D movement sensed by the two systems. We found that percent recurrence and determinism in signals could provide more information for a 
common movement generator source of the signal (combination of sinusoidal platform movements here). A signal is deterministic if there is no uncertainty with respect to its value at any instant of time. Recurrence plots can be generated which show for each moment in time, the times at which a phase space trajectory visits roughly the same area in the phase space (Fig. 3). We found that recurrence plots are sensitive to the change of signal properties in the course of time, and are affected by high-frequency noise. We performed RQA of the movement signals for 4 different frequencies and 4 different amplitude combinations of sway (Table 1). We found that the recurrence rate was highly dependent on noise and was affected by the noise in Mini-Logger, but determinism was found to equivalent to both the systems. This study validated the measured signals originated from a common source. However, our results revealed that stochastic resonance during these somatosensory-compromised conditions improved postural sway compared to control groups without vibratory input. These results are in agreement with previous research examining the effects of stochastic resonance on postural sway[2, 8, 15-22]. We found significant improvements in sway fluctuation measurements at the vibration frequency of $220 \mathrm{~Hz}$. These results suggest that there may be an ideal frequency at which stochastic resonance can be applied in order to promote good standing balance. These subtle changes in postural sway were identified by MiniLogger. Wearable sensors are widely being used for investigating fall risk assessment and fall predictions[23, 24] and are helpful to clinicians to identify patients with high fall risk.

\section{CONCLUSION}

This work contributes to the development of cheap wearable sensor, capable of measuring postural sway and assess fall risk. Our current findings show that IMU's can be readily designed and developed and used in measuring postural control. Wearable inertial sensors offer the opportunity of sensitive and objective balance assessments with high clinical feasibility due to small size, noninvasiveness, and portability. The work also contributes to developing novel methods for validation of sway sensed by two different systems. In conclusion, the development of cheap wearable systems can be utilized to prevent falls, fallrelated injuries, and thereby improve the quality of life of older adults and reduce medical costs related to falls.

\section{REFERENCES}

[1] A. Shumway-Cook and M. H. Woollacott, Motor Control: Translating Research Into Clinical Practice: Lippincott Williams \& Wilkins, 2007.

[2] M. Bagherzadeh Cham, M. A. Mohseni-Bandpei, M. Bahramizadeh, S. Kalbasi, and A. Biglarian, "The clinical and biomechanical effects of subthreshold random noise on the plantar surface of the foot in diabetic patients and elder people: A systematic review," Prosthet Orthot Int, vol. 40, pp. 658-667, Dec 2016.

[3] S. Nobusako, M. Osumi, A. Matsuo, T. Fukuchi, A. Nakai, T. Zama, et al., "Stochastic resonance improves visuomotor temporal integration in healthy young adults," PLoS One, vol. 13, p. e0209382, 2018.

[4] O. White, J. Babič, C. Trenado, L. Johannsen, and N. Goswami, "The Promise of Stochastic Resonance in Falls Prevention," Frontiers in Physiology, vol. 9, 2019.

[5] J. Zhou, L. Lipsitz, D. Habtemariam, and B. Manor, "Sub-sensory vibratory noise augments the physiologic complexity of postural control in older adults," J Neuroeng Rehabil, vol. 13, p. 44, May 32016.

[6] A. Aboutorabi, M. Arazpour, M. Bahramizadeh, F. Farahmand, and R. Fadayevatan, "Effect of vibration on postural control and gait of elderly subjects: a systematic review," Aging Clin Exp Res, vol. 30, pp. 713-726, Jul 2018.

[7] L. A. Lipsitz, M. Lough, J. Niemi, T. Travison, H. Howlett, and B. Manor, "A shoe insole delivering subsensory vibratory noise improves balance and gait in healthy elderly people," Arch Phys Med Rehabil, vol. 96, pp. 432-9, Mar 2015.

[8] D. G. Stephen, B. J. Wilcox, J. B. Niemi, J. R. Franz, D. Kerrigan, and S. E. D'Andrea, "Baseline-dependent effect of noiseenhanced insoles on gait variability in healthy elderly walkers," Gait Posture, vol. 36, pp. 537-40, Jul 2012.

[9] D. L. Miranda, W. H. Hsu, K. Petersen, S. Fitzgibbons, J. Niemi, N. Lesniewski-Laas, et al., "Sensory Enhancing Insoles Modify Gait during Inclined Treadmill Walking with Load," Med Sci Sports Exerc, vol. 48, pp. 860-8, May 2016.

[10] M. Dettmer, A. Pourmoghaddam, B. C. Lee, and C. S. Layne, "Associations between Tactile Sensory Threshold and Postural Performance and Effects of Healthy Aging and Subthreshold Vibrotactile Stimulation on Postural Outcomes in a Simple Dual Task," Curr Gerontol Geriatr Res, vol. 2016, p. 9797369, 2016.

[11] N. W. Hochreiter, M. J. Jewell, L. Barber, and P. Browne, "Effect of vibration on tactile sensitivity," Phys Ther, vol. 63, pp. 934-7, Jun 1983.

[12] S. Wallot and D. Mønster, "Calculation of Average Mutual Information (AMI) and False-Nearest Neighbors (FNN) for the Estimation of Embedding Parameters of Multidimensional Time Series in Matlab," Frontiers in Psychology, vol. 9, 2018.

[13] N. Marwan, M. Carmenromano, M. Thiel, and J. Kurths, "Recurrence plots for the analysis of complex systems," Physics Reports, vol. 438, pp. 237-329, 2007.

[14] C. L. Webber, Jr. and J. P. Zbilut, "Dynamical assessment of physiological systems and states using recurrence plot strategies," J Appl Physiol (1985), vol. 76, pp. 965-73, Feb 1994.

[15] R. Cloutier, S. Horr, J. B. Niemi, S. D'Andrea, C. Lima, J. D. Harry, et al., "Prolonged mechanical noise restores tactile sense in diabetic neuropathic patients," Int J Low Extrem Wounds, vol. 8, pp. 6-10, Mar 2009.

[16] L. Khaodhiar, J. B. Niemi, R. Earnest, C. Lima, J. D. Harry, and A. Veves, "Enhancing sensation in diabetic neuropathic foot with mechanical noise," Diabetes Care, vol. 26, pp. 3280-3, Dec 2003.

[17] Q. Wei, D.-H. Liu, K.-H. Wang, Q. Liu, M. Abbod, B. Jiang, et al., "Multivariate Multiscale Entropy Applied to Center of Pressure Signals Analysis: An Effect of Vibration Stimulation of Shoes," Entropy, vol. 14, pp. 2157-2172, 2012.

[18] C. C. Wang and W. H. Yang, "Using detrended fluctuation analysis (DFA) to analyze whether vibratory insoles enhance balance stability for elderly fallers," Arch Gerontol Geriatr, vol. 55, pp. 673-6, Nov-Dec 2012.

[19] A. M. Galica, H. G. Kang, A. A. Priplata, S. E. D'Andrea, O. V. Starobinets, F. A. Sorond, et al., "Subsensory vibrations to the feet reduce gait variability in elderly fallers," Gait Posture, vol. 30, pp. 383-7, Oct 2009.

[20] J. M. Hijmans, J. H. Geertzen, W. Zijlstra, A. L. Hof, and K. Postema, "Effects of vibrating insoles on standing balance in diabetic neuropathy," J Rehabil Res Dev, vol. 45, pp. 1441-9, 2008.

[21] A. A. Priplata, B. L. Patritti, J. B. Niemi, R. Hughes, D. C. Gravelle, L. A. Lipsitz, et al., "Noise-enhanced balance control in patients with diabetes and patients with stroke," Ann Neurol, vol. 59, pp. 4-12, Jan 2006.

[22] A. A. Priplata, J. B. Niemi, J. D. Harry, L. A. Lipsitz, and J. J. Collins, "Vibrating insoles and balance control in elderly people," Lancet, vol. 362, pp. 1123-4, Oct 42003.

[23] T. Lockhart, C. Frame, R. Soangra, and J. Lach, "Fall risk prediction using wearable wireless sensors," SPIE Newsroom, 2014.

[24] C. Frames, R. Soangra, T. Lockhart, J. Lach, D. Ha, K. Roberto, et al., "Dynamical Properties of Postural Control in Obese Community-Dwelling Older Adults," Sensors, vol. 18, p. 1692, 2018. 